\title{
THE GLOBAL CHARACTER OF SOLUTIONS OF AN ANTI-COMPETITIVE SYSTEM OF RATIONAL DIFFERENCE EQUATIONS
}

\author{
CHRIS D. LYND
}

This paper is dedicated to Professor Mustafa Kulenović, an outstanding teacher and mentor, in honor of his $60^{\text {th }}$ birthday

\begin{abstract}
In this paper, we analyze the global character of the solutions of an anti-competitive system of rational difference equations. We prove that the solutions of the system can have three different types of global behavior, corresponding to different regions of the parameter space. Our analysis utilizes a global convergence theorem from Camouzis and Ladas, and two theorems from Kulenović and Merino that apply to competitive systems.
\end{abstract}

\section{INTRODUCTION}

Consider the class of systems of difference equations of the form

$$
\left.\begin{array}{l}
x_{n+1}=\frac{\alpha_{1}+\beta_{1} x_{n}+\gamma_{1} y_{n}}{A_{1}+B_{1} x_{n}+C_{1} y_{n}} \\
y_{n+1}=\frac{\alpha_{2}+\beta_{2} x_{n}+\gamma_{2} y_{n}}{A_{2}+B_{2} x_{n}+C_{2} y_{n}}
\end{array}\right\} \text { for } n=0,1,2, \ldots
$$

where the parameters and the initial terms, $x_{0}$ and $y_{0}$, are non-negative numbers such that the denominators are never equal to zero.

In 2008, Camouzis, Kulenović, Ladas, and Merino [1] introduced a numbering system which divided the class of systems in (1.1) into 2,401 separate systems, where each system of equations has a unique combination of positive parameters.

2000 Mathematics Subject Classification. 39A10, 39A11.

Key words and phrases. Difference equations, competitive maps, anti-competitive maps, global asymptotic stability, period-2 solutions. 
Since that time, many papers have been written [5]-[16] analyzing the boundedness, and the global behavior, of the solutions of systems of equations of the form in (1.1).

Using the numbering system introduced in $[1]$, system \# $(16,18)$ contains the six positive parameters shown below.

$$
\left.\begin{array}{l}
x_{n+1}=\frac{\gamma_{1} y_{n}}{A_{1}+B_{1} x_{n}} \\
y_{n+1}=\frac{\beta_{2} x_{n}}{B_{2} x_{n}+C_{2} y_{n}}
\end{array}\right\} \text { for } n=0,1,2, \ldots
$$

In this paper, we analyze the global character of the solutions of system (1.2), where the parameters are positive numbers and the initial terms, $x_{0}$ and $y_{0}$, are non-negative numbers such that $x_{0}+y_{0}>0$.

\section{Preliminaries}

The following definitions and theorems will be used in this paper.

Definition 2.1. The symbol $\preceq_{S E}$ denotes the south-east partial ordering of the non-negative quadrant. That is, $(x, y) \preceq_{S E}(a, b)$ if and only if $x \leq a$ and $y \geq b$. A strict inequality is defined as $(x, y) \prec_{S E}(a, b)$ if $(x, y) \preceq_{S E}(a, b)$ and $(x, y) \neq(a, b)$. A strong inequality is defined as $(x, y) \prec \prec S E(a, b)$ if $x<a$ and $y>b$.

Suppose that $P_{1} \prec_{S E} P_{2}$. Then, we use the ordered interval $\llbracket P_{1}, P_{2} \rrbracket$ to denote the set $\left\{(x, y): P_{1} \preceq_{S E}(x, y) \preceq_{S E} P_{2}\right\}$.

Definition 2.2. Let $R$ be a subset of $\mathbb{R}^{2}$ with nonempty interior, and let $F: R \rightarrow R$ be a continuous map where $F(x, y)=(f(x, y), g(x, y))$.

(a) The map $F$ is competitive if $f(x, y)$ is non-decreasing in $x$ and nonincreasing in $y$, and $g(x, y)$ is non-increasing in $x$ and non-decreasing in $y$.

(b) The map $F$ is strongly competitive if $f(x, y)$ is increasing in $x$ and decreasing in $y$, and $g(x, y)$ is decreasing in $x$ and increasing in $y$.

(c) The map $F$ is anti-competitive if $f(x, y)$ is non-increasing in $x$ and non-decreasing in $y$, and $g(x, y)$ is non-decreasing in $x$ and nonincreasing in $y$.

An important property of competitive maps is that they preserve the south-east ordering; that is, if $F$ is a competitive map and $(x, y) \preceq_{S E}(a, b)$, then $F((x, y)) \preceq_{S E} F((a, b))$. The map $\mathrm{F}$ is strongly order preserving when $(x, y) \prec_{S E}(a, b)$ implies that $F((x, y)) \prec_{S E} F((a, b))$.

If $F$ is anti-competitive, then the south-east ordering is reversed; that is, if $(x, y) \preceq_{S E}(a, b)$, then $F((a, b)) \preceq_{S E} F((x, y))$. So, if the map $F: R \rightarrow R$ is 
anti-competitive, then the map $F^{2}: R \rightarrow R$, where $F^{2}(x, y)=F(F(x, y))$, is competitive and preserves the south-east ordering.

Theorem 2.1. (The Linearized Stability Theorem) Let I be an interval of real numbers and let $F: I \times I \rightarrow I \times I$ be a continuously differentiable map where $F(x, y)=(f(x, y), g(x, y))$. Let $E=(\bar{x}, \bar{y})$ be an equilibrium point of the system of difference equations

$$
\left.\begin{array}{l}
x_{n+1}=f\left(x_{n}, y_{n}\right) \\
y_{n+1}=g\left(x_{n}, y_{n}\right)
\end{array}\right\} \text { for } n=0,1,2, \ldots .
$$

Let $J_{F}(E)=\left[\begin{array}{ll}f_{x}(E) & f_{y}(E) \\ g_{x}(E) & g_{y}(E)\end{array}\right]$ be the Jacobian matrix of $F$, evaluated at $E$.

Then, the following statements are true.

(a) If both eigenvalues of $J_{F}(E)$ are inside the unit circle, that is, if

$$
\left|\operatorname{Tr}\left(J_{F}(E)\right)\right|<1+\operatorname{Det}\left(J_{F}(E)\right)<2,
$$

then $E$ is locally asymptotically stable.

(b) If both eigenvalues of $J_{F}(E)$ are outside the unit circle, that is, if

$$
\left|\operatorname{Det}\left(J_{F}(E)\right)\right|>1 \text { and }\left|\operatorname{Tr}\left(J_{F}(E)\right)\right|<\left|1+\operatorname{Det}\left(J_{F}(E)\right)\right| \text {, }
$$

then $E$ is a repeller.

(c) If one eigenvalue of $J_{F}(E)$ is inside the unit circle and one eigenvalue is outside the unit circle, that is, if

$$
\left|\operatorname{Tr}\left(J_{F}(E)\right)\right|>\left|1+\operatorname{Det}\left(J_{F}(E)\right)\right|
$$

and

$$
\left(\operatorname{Tr}\left(J_{F}(E)\right)\right)^{2}-4 \cdot \operatorname{Det}\left(J_{F}(E)\right)>0
$$

then $E$ is a saddle point.

(d) If at least one eigenvalue of $J_{F}(E)$ is on the unit circle, that is, if

$$
\left|\operatorname{Tr}\left(J_{F}(E)\right)\right|=\left|1+\operatorname{Det}\left(J_{F}(E)\right)\right|
$$

or

$$
\operatorname{Det}\left(J_{F}(E)\right)=1 \text { and } \operatorname{Tr}\left(J_{F}(E)\right) \leq 2,
$$

then $E$ is non-hyperbolic.

Theorem 2.2. (Camouzis and Ladas [2]) Let I be a set of real numbers and let

$$
f: I \times I \rightarrow I
$$

be a function $f(u, v)$, which decreases in $u$ and increases in $v$.

Then, for every solution $\left\{x_{n}\right\}_{n=-1}^{\infty}$ of the equation

$$
x_{n+1}=f\left(x_{n}, x_{n-1}\right), n=0,1,2, \ldots
$$

the subsequences $\left\{x_{2 n}\right\}_{n=0}^{\infty}$ and $\left\{x_{2 n-1}\right\}_{n=0}^{\infty}$ are eventually monotonic. 
The next two theorems will be used in the case where we have a strongly competitive, strongly order preserving map from $\mathbb{R}_{+}^{2}$ to $\mathbb{R}_{+}^{2}$. For simplification purposes, the theorems are not stated in their full generality.

Theorem 2.3. (Kulenović and Merino [3]) Let $u_{1} \prec u_{2}$ be fixed points of a strongly order preserving, continuous map $F: A \rightarrow A$, where $A \subset \mathbb{R}^{2}$ and $\llbracket u_{1}, u_{2} \rrbracket \subset A$. Suppose there are no fixed points of $F$ in $\llbracket u_{1}, u_{2} \rrbracket$ other than $u_{1}$ and $u_{2}$.

Then, the interior of $\llbracket u_{1}, u_{2} \rrbracket$ is either a subset of the basin of attraction of $u_{1}$ or is a subset of the basin of attraction of $u_{2}$.

Theorem 2.4. (Kulenović and Merino [4]) Let $F$ be a strongly competitive map on a rectangular region $R \subset \mathbb{R}^{2}$ that contains a fixed point $P$ in its interior. Suppose that $F$ has a $C^{1}$ extension to a neighborhood of $P$. Suppose also that the Jacobian matrix of $F$ at $P$ has real eigenvalues, $\lambda$ and $\mu$, such that $0<|\lambda|<\mu,|\lambda|<1$, and the eigenspace $E^{\lambda}$, corresponding to $\lambda$, is not a coordinate axis.

Then, there exists a curve $C \subset R$ through $P$ that is invariant under $F$ and is a subset of the basin of attraction of $P$, such that $C$ is tangential to the eigenspace $E^{\lambda}$ at $P$, and $C$ is a strictly increasing continuous function of the first coordinate on an interval. Any endpoints of $C$ in the interior of $R$ are either fixed points, or minimal period-2 points, of the map $F$.

\section{LOCAL STABILITY ANALYSIS}

The system of equations in (1.2) can be written in normalized form as

$$
\left.\begin{array}{l}
x_{n+1}=\frac{y_{n}}{1+x_{n}} \\
y_{n+1}=\frac{\beta_{2} x_{n}}{B_{2} x_{n}+y_{n}}
\end{array}\right\} \text { for } n=0,1,2, \ldots
$$

where the parameters are positive numbers and the initial terms, $x_{0}$ and $y_{0}$, are non-negative numbers such that $x_{0}+y_{0}>0$.

3.1. Boundedness of solutions of the system. Let $\left\{x_{n}, y_{n}\right\}$ be a solution of system (3.1). Then $y_{n+1} \leq \frac{\beta_{2}}{B_{2}}$ for all $n \geq 0$. Thus, $x_{n+1}<y_{n} \leq \frac{\beta_{2}}{B_{2}}$ for all $n \geq 1$. So, every solution of system (3.1) is bounded and there is a uniform bound for all solutions.

3.2. Equilibrium points. Solving the system of equilibrium equations corresponding to system (3.1), one can see that $\bar{y}=\bar{x}^{2}+\bar{x}$ and that $\bar{x}$ is a solution of the equation

$$
\bar{x}^{3}+\left(2+B_{2}\right) \bar{x}^{2}+\left(1+B_{2}\right) \bar{x}-\beta_{2}=0 .
$$


By DeCartes' Rule of Signs, equation (3.2) has a unique positive solution, and thus, there is a unique positive equilibrium point of system (3.1).

Let $F$ be the map corresponding to system (3.1). That is, let

$$
F\left(\begin{array}{l}
x \\
y
\end{array}\right)=\left(\begin{array}{c}
\frac{y}{1+x} \\
\frac{\beta_{2} x}{B_{2} x+y}
\end{array}\right)
$$

Then, the Jacobian matrix of $F$ is $J_{F}=\left[\begin{array}{cc}\frac{-y}{(1+x)^{2}} & \frac{1}{1+x} \\ \frac{\beta_{2} y}{\left(B_{2} x+y\right)^{2}} & \frac{-\beta_{2} x}{\left(B_{2} x+y\right)^{2}}\end{array}\right]$.

Using the equilibrium equations

$$
\bar{x}=\frac{\bar{y}}{1+\bar{x}}
$$

and

$$
\bar{y}=\frac{\beta_{2} \bar{x}}{B_{2} \bar{x}+\bar{y}},
$$

one can evaluate the Jacobian matrix at the equilibrium point to get

$$
J_{F}(\bar{x}, \bar{y})=\left[\begin{array}{cc}
\frac{-\bar{x}^{2}}{\bar{y}} & \bar{x} \\
\overline{\bar{y}} & \frac{-\bar{y}^{2}}{\beta_{2} \bar{x}}
\end{array}\right] .
$$

The characteristic equation for this matrix is

$$
\lambda^{2}+\frac{\beta_{2} \bar{x}^{3}+\bar{y}^{3}}{\beta_{2} \bar{x} \bar{y}} \lambda+\frac{\bar{x}^{2} \bar{y}-\bar{y}^{2}}{\beta_{2} \bar{x}}=0 .
$$

Using the Linearized Stability Theorem 2.1, we see that both eigenvalues of the matrix in (3.6) lie inside the unit circle, if and only if,

$$
\left|\frac{-\beta_{2} \bar{x}^{3}-\bar{y}^{3}}{\beta_{2} \bar{x} \bar{y}}\right|<1+\frac{\bar{y}\left(\bar{x}^{2}-\bar{y}\right)}{\beta_{2} \bar{x}}<2 .
$$

We will show that the inequality in (3.7) is true, if and only if, $\beta_{2}<$ $B_{2}{ }^{2}-B_{2}$. Once this fact is established, the remaining part of the local stability analysis will be straight-forward.

Using the equation in (3.4), we see that $\bar{x}^{2}-\bar{y}=-\bar{x}$, and so the inequality in (3.7) is true, if and only if,

$$
\frac{\beta_{2} \bar{x}^{3}+\bar{y}^{3}}{\beta_{2} \bar{x} \bar{y}}<1-\frac{\bar{y}}{\beta_{2}}<2 .
$$

Since $\bar{y}$ and $\beta_{2}$ are positive, it follows that $1-\frac{\bar{y}}{\beta_{2}}<2$. 
So, the inequality in (3.7) is true, if and only if,

$$
\beta_{2} \bar{x}^{3}+\bar{y}^{3}<\beta_{2} \bar{x} \bar{y}-\bar{x} \bar{y}^{2} .
$$

Using the equation in (3.4), we see that $\bar{y}=\bar{x}^{2}+\bar{x}$, and so

$$
\beta_{2} \bar{x}^{3}+\bar{y}^{2}\left(\bar{x}^{2}+\bar{x}\right)<\beta_{2} \bar{x}^{3}+\beta_{2} \bar{x}^{2}-\bar{x} \bar{y}^{2} .
$$

So,

$$
\bar{y}^{2}\left(\bar{x}^{2}+2 \bar{x}\right)<\beta_{2} \bar{x}^{2} .
$$

Dividing both sides of the inequality by $\bar{x} \bar{y}$, we get

$$
\bar{y}(\bar{x}+2)<\frac{\beta_{2} \bar{x}}{\bar{y}} .
$$

Using the equation in (3.5), we see that $\frac{\beta_{2} \bar{x}}{\bar{y}}=B_{2} \bar{x}+\bar{y}$. So,

$$
\bar{x}(\bar{x}+1)(\bar{x}+2)<B_{2} \bar{x}+\bar{y} .
$$

Dividing both sides of the inequality by $\bar{x}$, we get

$$
\bar{x}^{2}+3 \bar{x}+2<B_{2}+\bar{x}+1 .
$$

Solving this quadratic inequality yields,

$$
0<\bar{x}<\sqrt{B_{2}}-1
$$

where $B_{2}>1$.

Let $p(x)=x^{3}+\left(2+B_{2}\right) x^{2}+\left(1+B_{2}\right) x-\beta_{2}$, which is the function corresponding to the equilibrium equation in (3.2).

Since $p(\bar{x})=0$, we have $\bar{x}<\sqrt{B_{2}}-1$, if and only if, $p\left(\sqrt{B_{2}}-1\right)>0$, which is true, if and only if, $\beta_{2}<B_{2}^{2}-B_{2}$.

Using the Linearized Stability Theorem 2.1, we see that the equilibrium point of system (3.1) is locally asymptotically stable if $\beta_{2}<B_{2}{ }^{2}-B_{2}$, it is a saddle point if $\beta_{2}>B_{2}{ }^{2}-B_{2}$, and it is non-hyperbolic if $\beta_{2}=B_{2}{ }^{2}-B_{2}$.

In the case where $\beta_{2}=B_{2}^{2}-B_{2}$, we have $(\bar{x}, \bar{y})=\left(\sqrt{B_{2}}-1, B_{2}-\sqrt{B_{2}}\right)$. Also, $\mu=-1$ is an eigenvalue of the matrix $J_{F}(\bar{x}, \bar{y})$ in (3.6). If $\lambda$ is the other eigenvalue, then $\mu \cdot \lambda=\frac{\bar{x}^{2} \bar{y}-\bar{y}^{2}}{\beta_{2} \bar{x}}$, and so we have $|\lambda|<1$.

3.3. Period-2 solutions. Suppose that there is a minimal period-2 solution $\left\{\left(v_{1}, w_{1}\right),\left(v_{2}, w_{2}\right),\left(v_{1}, w_{1}\right), \ldots\right\}$ of system (3.1). Then, the four equations below must be satisfied.

$$
\begin{array}{rlrl}
v_{1} & =\frac{w_{2}}{1+v_{2}} & v_{2} & =\frac{w_{1}}{1+v_{1}} \\
w_{1}=\frac{\beta_{2} v_{2}}{B_{2} v_{2}+w_{2}} & \text { and } & w_{2}=\frac{\beta_{2} v_{1}}{B_{2} v_{1}+w_{1}}
\end{array}
$$


Using the equations for $v_{1}$ and $v_{2}$ in system (3.8), it follows that

$$
v_{1}-v_{2}=w_{2}-w_{1} \text {. }
$$

So, $v_{1}=v_{2}$, if and only if, $w_{1}=w_{2}$. Thus, if system (3.1) has a minimal period-2 solution, then $v_{1} \neq v_{2}$ and $w_{1} \neq w_{2}$.

Using the same algebraic steps with the equations for $w_{1}$ and $w_{2}$ in system (3.8), one sees that

$$
B_{2}\left(v_{2} w_{1}-v_{1} w_{2}\right)=\beta_{2}\left(v_{2}-v_{1}\right)
$$

So,

$$
B_{2}\left(v_{2} w_{1}-v_{1} w_{1}+v_{1} w_{1}-v_{1} w_{2}\right)=\beta_{2}\left(v_{2}-v_{1}\right)
$$

and so,

$$
B_{2}\left(w_{1}\left(v_{2}-v_{1}\right)+v_{1}\left(w_{1}-w_{2}\right)\right)=\beta_{2}\left(v_{2}-v_{1}\right) .
$$

Using the equation in (3.9), we get

$$
B_{2}\left(w_{1}\left(v_{2}-v_{1}\right)+v_{1}\left(v_{2}-v_{1}\right)\right)=\beta_{2}\left(v_{2}-v_{1}\right) .
$$

We are assuming that $v_{1} \neq v_{2}$, so

$$
B_{2}\left(w_{1}+v_{1}\right)=\beta_{2} \text {. }
$$

Therefore, if there is a minimal period-2 point of system (3.1), it must be a point on the line segment

$$
w_{1}=-v_{1}+\frac{\beta_{2}}{B_{2}} \text { with } 0 \leq v_{1} \leq \frac{\beta_{2}}{B_{2}} .
$$

For all positive parameters, the endpoints of line segment (3.10), that is $\left(0, \frac{\beta_{2}}{B_{2}}\right)$ and $\left(\frac{\beta_{2}}{B_{2}}, 0\right)$, are minimal period-2 points of system (3.1).

The remaining points on line segment (3.10) are period-2 points of system (3.1), if and only if, $B_{2}>1$ and $\beta_{2}=B_{2}{ }^{2}-B_{2}$.

Using the map $F$ in (3.3), one can evaluate the Jacobian matrix of $F^{2}$ at the point $\left(0, \frac{\beta_{2}}{B_{2}}\right)$ to get

$$
J_{F^{2}}\left(0, \frac{\beta_{2}}{B_{2}}\right)=\left[\begin{array}{cc}
\frac{B_{2}{ }^{2}}{B_{2}+\beta_{2}} & 0 \\
-1 & 0
\end{array}\right] .
$$

For the Jacobian matrix in (3.11), one eigenvalue is zero, and the other eigenvalue is inside the unit circle, if and only if, $\beta_{2}>B_{2}{ }^{2}-B_{2}$. This is also true for the point $\left(\frac{\beta_{2}}{B_{2}}, 0\right)$.

Using the Linearized Stability Theorem 2.1, we see that the points $\left(0, \frac{\beta_{2}}{B_{2}}\right)$ and $\left(\frac{\beta_{2}}{B_{2}}, 0\right)$ are locally asymptotically stable fixed points of $F^{2}$ if $\beta_{2}>$ 
$B_{2}{ }^{2}-B_{2}$, they are saddle points of $F^{2}$ if $\beta_{2}<B_{2}{ }^{2}-B_{2}$, and they are non-hyperbolic if $\beta_{2}=B_{2}{ }^{2}-B_{2}$.

In the case where $\beta_{2}=B_{2}{ }^{2}-B_{2}$, every point on the line segment in (3.10) is a period-2 point of $F$.

\section{Global stability analysis}

We can decouple the equations in system (3.1) and arrive at the $2^{\text {nd }}$-order difference equation

$$
x_{n+1}=\frac{\beta_{2} x_{n-1}}{x_{n}+x_{n}^{2}+x_{n-1}\left(B_{2}+\left(1+B_{2}\right) x_{n}+x_{n}^{2}\right)} \text { for } n=1,2,3, \ldots
$$

where the parameters are positive numbers and the initial terms, $x_{0}$ and $x_{1}$, are nonnegative numbers such that $x_{0}+x_{1}>0$.

Let $f$ be the function corresponding to difference equation (4.1), which is

$$
f(u, v)=\frac{\beta_{2} v}{u+u^{2}+B_{2} v+\left(1+B_{2}\right) u v+u^{2} v} .
$$

Let $\left\{x_{n}\right\}_{n=0}^{\infty}$ be a solution of difference equation (4.1). Then $x_{n} \in\left[0, \frac{\beta_{2}}{B_{2}}\right]$ for all $n \geq 2$. Furthermore, if $(u, v) \in\left[0, \frac{\beta_{2}}{B_{2}}\right] \times\left[0, \frac{\beta_{2}}{B_{2}}\right]$, then $f(u, v) \in$ $\left[0, \frac{\beta_{2}}{B_{2}}\right]$.

By examining the partial derivatives of $f$, we find that $f$ is decreasing in $u$ and is increasing in $v$. By Theorem 2.2, the subsequences $\left\{x_{2 n}\right\}$ and $\left\{x_{2 n+1}\right\}$ of the solution of difference equation (4.1) are eventually monotonic. Since every solution is bounded, $\left\{x_{2 n}\right\}$ and $\left\{x_{2 n+1}\right\}$ converge.

Let $\left\{x_{n}, y_{n}\right\}_{n=0}^{\infty}$ be a solution of system (3.1) where $x_{0}$ and $y_{0}$ are nonnegative numbers such that $x_{0}+y_{0}>0$.

Then, $\left\{x_{n}\right\}_{n=0}^{\infty}$ is the solution of difference equation (4.1) where $x_{1}=$ $\frac{y_{0}}{1+x_{0}}$.

Since $\left\{x_{2 n}\right\}_{n=0}^{\infty}$ and $\left\{x_{2 n+1}\right\}_{n=0}^{\infty}$ converge, let

$$
\lim _{n \rightarrow \infty} x_{2 n}=L_{1} \text { and } \lim _{n \rightarrow \infty} x_{2 n+1}=L_{2} .
$$

Using the first equation in system (3.1), we see that for all $n \geq 0$,

$$
y_{2 n}=x_{2 n+1}\left(1+x_{2 n}\right) \text { and } y_{2 n+1}=x_{2 n+2}\left(1+x_{2 n+1}\right) .
$$

And so we have,

$$
\lim _{n \rightarrow \infty} y_{2 n}=L_{2}\left(1+L_{1}\right) \text { and } \lim _{n \rightarrow \infty} y_{2 n+1}=L_{1}\left(1+L_{2}\right) .
$$

This proves Theorem 4.1 below. 
Theorem 4.1. Let $\left\{x_{n}, y_{n}\right\}$ be a solution of the system of difference equations in (3.1). Then, there are non-negative numbers $L_{1}$ and $L_{2}$, such that $\left\{\left(x_{2 n}, y_{2 n}\right)\right\} \rightarrow\left(L_{1}, L_{2}\left(1+L_{1}\right)\right)$ and $\left\{\left(x_{2 n+1}, y_{2 n+1}\right)\right\} \rightarrow\left(L_{2}, L_{1}\left(1+L_{2}\right)\right)$.

Some solutions of system (3.1) converge. For these solutions, there is an $L_{1}$ such that $\left\{\left(x_{n}, y_{n}\right)\right\} \rightarrow\left(L_{1}, L_{1}+L_{1}^{2}\right)$.

We will provide a detailed analysis of the global behavior of the solutions of system (3.1) by analyzing different regions of the paramter space. The picture below illustrates the regions we will analyze and the global behavior of the solutions in each region.

$$
\left.\begin{array}{l}
x_{n+1}=\frac{y_{n}}{1+x_{n}} \\
y_{n+1}=\frac{\beta_{2} x_{n}}{B_{2} x_{n}+y_{n}}
\end{array}\right\} \text { for } n=0,1,2, \ldots
$$

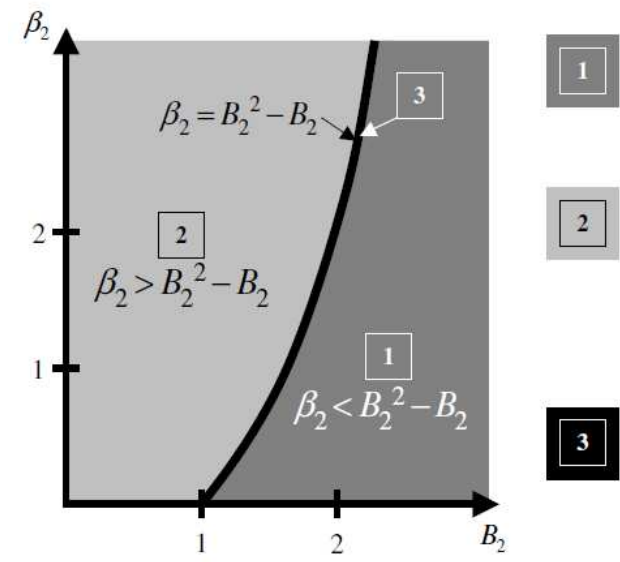

If $\beta_{2}<B_{2}{ }^{2}-B_{2}$, then almost every solution converges to $(\bar{x}, \bar{y})$.

If $\beta_{2}>B_{2}^{2}-B_{2}$, then almost every solution converges to the period-2 solution $\left\{\left(0, \frac{\beta_{2}}{B_{2}}\right),\left(\frac{\beta_{2}}{B_{2}}, 0\right),\left(0, \frac{\beta_{2}}{B_{2}}\right), \ldots\right\}$

If $\beta_{2}=B_{2}{ }^{2}-B_{2}$, then every solution converges to a period-2 solution on the line $y=-x+\frac{\beta_{2}}{B_{2}}$.

Let $\left\{x_{n}, y_{n}\right\}$ be a solution of the system of difference equations in (3.1) and let $F$ be the map in (3.3).

We begin by examining the case where the initial term $\left(x_{0}, y_{0}\right)$ is on an axis. Notice that

$$
F\left(\begin{array}{c}
x_{0} \\
0
\end{array}\right)=\left(\begin{array}{c}
0 \\
\frac{\beta_{2}}{B_{2}}
\end{array}\right) \text { and } F\left(\begin{array}{c}
0 \\
\frac{\beta_{2}}{B_{2}}
\end{array}\right)=\left(\begin{array}{c}
\frac{\beta_{2}}{B_{2}} \\
0
\end{array}\right) .
$$

Also,

$$
F\left(\begin{array}{c}
0 \\
y_{0}
\end{array}\right)=\left(\begin{array}{c}
y_{0} \\
0
\end{array}\right) \text { and } F\left(\begin{array}{c}
y_{0} \\
0
\end{array}\right)=\left(\begin{array}{c}
0 \\
\frac{\beta_{2}}{B_{2}}
\end{array}\right) .
$$

So, if $\left(x_{0}, y_{0}\right)$ is on the positive $x$-axis or the positive $y$-axis, the solution of system (3.1) becomes the minimal period-2 solution on the axes by at least the second iterate of $F$. 
For the remainder of the analysis, we will consider the case where both $x_{0}$ and $y_{0}$ are positive.

Let the interval $I=\left(0, \frac{\beta_{2}}{B_{2}}\right)$. Since $\left(x_{n}, y_{n}\right) \in I \times I$ for all $n \geq 2$, we need only consider solutions of system (3.1) that are in this rectangle.

The map $F: I \times I \rightarrow I \times I$ is anti-competitive. The map $F^{2}: I \times I \rightarrow I \times I$ is strongly competitive and stongly preserves the south-east ordering.

By Theorem 4.1, we know that $\left\{x_{2 n}\right\},\left\{y_{2 n}\right\},\left\{x_{2 n+1}\right\}$ and $\left\{y_{2 n+1}\right\}$ converge. Thus, if we begin at any $\left(x_{0}, y_{0}\right) \in I \times I$, the iterates of $F^{2}$ will converge.

Let $E$ be the equilibrium point of system (3.1). Let $P_{1}$ and $P_{2}$ be the minimal period-2 points of system (3.1) that are on the axes; that is, let $P_{1}=\left(0, \frac{\beta_{2}}{B_{2}}\right)$ and let $P_{2}=\left(\frac{\beta_{2}}{B_{2}}, 0\right)$.

Region 1: Let $\beta_{2}<B_{2}{ }^{2}-B_{2}$.

In this region of parameter space, the equilibrium point $E$ is located in the region bounded by the line segment in (3.10), the line $y=x$, and the line $y=\frac{\beta_{2}}{B_{2}}$. The eigenvalues of $J_{F}(E), \lambda$ and $\mu$, are inside the unit circle. Thus, $\lambda^{2}$ and $\mu^{2}$, the eigenvalues of $J_{F^{2}}(E)$, are also inside the unit circle. Therefore, $E$ is a locally asymptotically stable fixed point of the map $F^{2}$.

The minimal period-2 points of system (3.1), $P_{1}$ and $P_{2}$, are saddle points of the map $F^{2}$. Furthermore, $E, P_{1}$, and $P_{2}$ are the only fixed points of $F^{2}$.

The picture below illustrates the iterates of $F^{2}$ when $\beta_{2}<B_{2}{ }^{2}-B_{2}$.

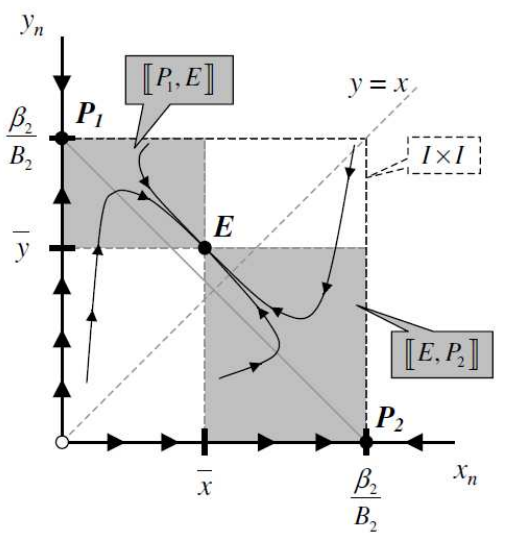

Since $E$ is a locally asymptotically stable fixed point of $F^{2}$, there are some points in the interior of $\llbracket P_{1}, E \rrbracket$, and some points in the interior of $\llbracket E, P_{2} \rrbracket$, that are in the basin of attraction of $E$. Thus, by Theorem 2.3, the interior of $\llbracket P_{1}, E \rrbracket \cup \llbracket E, P_{2} \rrbracket$ is in the basin of attraction of $E$. 
Suppose that $\left(x_{0}, y_{0}\right)$ is inside the rectangle $I \times I$, but is not in the interior of $\llbracket P_{1}, E \rrbracket \cup \llbracket E, P_{2} \rrbracket$.

Then, there is a point $\left(x_{L}, y_{L}\right)$ in the interior of $\llbracket P_{1}, E \rrbracket$, and a point $\left(x_{U}, y_{U}\right)$ in the interior of $\llbracket E, P_{2} \rrbracket$, such that $\left(x_{L}, y_{L}\right) \preceq_{S E}\left(x_{0}, y_{0}\right) \preceq_{S E}$ $\left(x_{U}, y_{U}\right)$. Since $F^{2}$ is competitive, it preserves the south-east ordering, so we have $F^{2 n}\left(x_{L}, y_{L}\right) \preceq_{S E} F^{2 n}\left(x_{0}, y_{0}\right) \preceq_{S E} F^{2 n}\left(x_{U}, y_{U}\right)$ for all $n \geq 1$. We know that $\left\{F^{2 n}\left(x_{L}, y_{L}\right)\right\}$ and $\left\{F^{2 n}\left(x_{U}, y_{U}\right)\right\}$ both converge to $E$, so $\left\{F^{2 n}\left(x_{0}, y_{0}\right)\right\}$ must also converge to $E$.

Since $\left(x_{0}, y_{0}\right) \in I \times I$, we know that $\left(x_{1}, y_{1}\right) \in I \times I$. Using the argument above, we see that $\left\{F^{2 n}\left(x_{1}, y_{1}\right)\right\}$ also converges to $E$.

Therefore, in Region 1 of the parameter space, if $x_{0}$ and $y_{0}$ are positive, the solution of system (3.1) converges to the unique equilibrium point $E$.

Region 2: Let $\beta_{2}>B_{2}{ }^{2}-B_{2}$.

In this region of parameter space, the equilibrium point $E$ is located in the region bounded by the line segment in (3.10), the line $y=x$, and the $y$-axis. The eigenvalues of $J_{F}(E), \lambda$ and $\mu$, are such that $|\lambda|<1$ and $|\mu|>1$. So we have $0<\left|\lambda^{2}\right|<1$ and $\left|\mu^{2}\right|>1$, which implies that $E$ is a saddle point of the map $F^{2}$.

If $\vec{v}$ is a non-zero vector that is parallel to an axis, then for any value of $\lambda, \vec{v} \notin \operatorname{Nul}\left(J_{F}(E)-\lambda I\right)$. So, the eigenvectors of $J_{F}(E)$ are not parallel to either axis. Since the eigenvectors of $J_{F}(E)$ are the eigenvectors of $\left(J_{F}(E)\right)^{2}=J_{F^{2}}(E)$, the eigenvectors of $J_{F^{2}}(E)$ are not parallel to either axis.

The minimal period-2 points of system (3.1), $P_{1}$ and $P_{2}$, are locally asymptotically stable fixed points of the map $F^{2}$. Furthermore, the points $E, P_{1}$, and $P_{2}$ are the only fixed points of $F^{2}$.

The picture below illustrates the iterates of $F^{2}$ when $\beta_{2}>B_{2}{ }^{2}-B_{2}$.

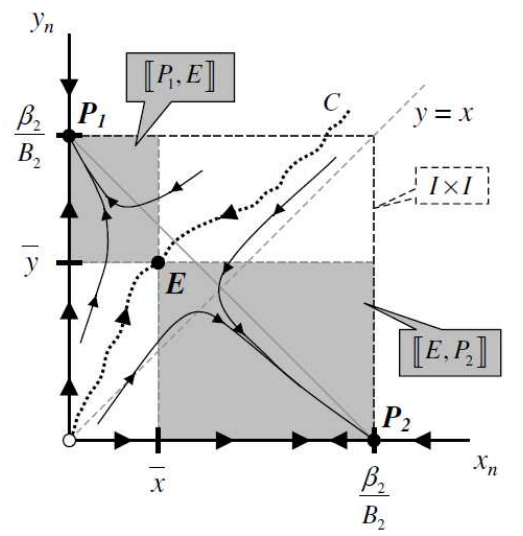


By Theorem 2.4, there is an increasing curve $C$ through $E$ that is invariant under $F^{2}$ and is a subset of the basin of attraction of $E$. This curve extends indefinitely to the north-east of $E$ because there are no prime period-2 points of $F^{2}$, and there are no fixed points of $F^{2}$ to the north-east of $E$.

This curve also extends from the south-west of $E$ to the boundary of the region $\left[0, \frac{\beta_{2}}{B_{2}}\right] \times\left[0, \frac{\beta_{2}}{B_{2}}\right]$. We know that if $\left(x_{n}, y_{n}\right)$ is a point on either positive axis, then the iterates of $F$, beginning at $\left(x_{n}, y_{n}\right)$, will converge to the period-2 points on the axes. Thus, the curve $C$ extends from the southwest of $E$ and has the point $(0,0)$ as its limiting point (note that the map $F^{2}$ is undefined at the origin).

Without loss of generality, suppose that $\left(x_{0}, y_{0}\right)$ is above the curve $C$. Then there is a point $\left(x_{C}, y_{C}\right)$ that is on curve $C$ and to the south-east of $\left(x_{0}, y_{0}\right)$.

Since the iterates of the map $F^{2}$, beginning at $\left(x_{C}, y_{C}\right)$, are on curve $C$, and the map $F^{2}$ strongly preserves the south-east ordering, all of the points in the subsequence $\left\{\left(x_{2 n}, y_{2 n}\right)\right\}$, beginning at $\left(x_{0}, y_{0}\right)$, are above curve $C$. We know that $\left\{\left(x_{2 n}, y_{2 n}\right)\right\}$ converges, so it must converge to either $E$ or $P_{1}$.

Furthermore, since $F$ is anti-competitive, $F\left(x_{C}, y_{C}\right) \prec_{S E} F\left(x_{0}, y_{0}\right)=$ $\left(x_{1}, y_{1}\right)$. Thus, all of the points in the subsequence $\left\{\left(x_{2 n+1}, y_{2 n+1}\right)\right\}$, beginning at $\left(x_{1}, y_{1}\right)$, are below curve $C$ and converge to either $E$ or $P_{2}$.

For the sake of contradiction, suppose that $\left\{\left(x_{2 n}, y_{2 n}\right)\right\}$ converges to $E$. Then, since $E$ is a saddle point of $F^{2}$ and $\left\{F^{2 n}\left(x_{C}, y_{C}\right)\right\}$ converges to $E$, there is some $k$ such that $F^{2 k}\left(x_{0}, y_{0}\right)$ and $F^{2 k}\left(x_{C}, y_{C}\right)$ are both on the local stable manifold through $E$, which is a strictly increasing curve. This would contradict the fact that the map $F^{2}$ preserves the south-east ordering. Thus, $\left\{\left(x_{2 n}, y_{2 n}\right)\right\}$ must converge to $P_{1}$.

By a similar argument, $\left\{\left(x_{2 n+1}, y_{2 n+1}\right)\right\}$ converges to $P_{2}$.

Therefore, in Region 2 of the parameter space, whenever $\left(x_{0}, y_{0}\right)$ is not on curve $C$, the solution of system (3.1) converges to the minimal period-2 solution on the axes, where the points in the subsequence $\left\{\left(x_{2 n}, y_{2 n}\right)\right\}$ are on one side of curve $C$, and the points in the subsequence $\left\{\left(x_{2 n+1}, y_{2 n+1}\right)\right\}$ are on the other side of curve $C$.

Region 3: Let $B_{2}>1$ and $\beta_{2}=B_{2}^{2}-B_{2}$.

In this region of parameter space, every point on the line segment in (3.10) is a period-2 point of system (3.1), and thus, is a fixed point of $F^{2}$. The equilibrium point $E=(\bar{x}, \bar{y})=\left(\sqrt{B_{2}}-1, B_{2}-\sqrt{B_{2}}\right)$ is on this line segment.

The eigenvalues of $J_{F}(E)$ are $\mu=-1$ and $0<\lambda=\frac{\bar{y}^{2}-\bar{x}^{2} \bar{y}}{\beta_{2} \bar{x}}<1$. So we have $0<\left|\lambda^{2}\right|<1=\mu^{2}$, which implies that $E$ is a non-hyperbolic fixed point of $F^{2}$. In addition, the eigenvectors of $J_{F^{2}}(E)$ are the same as the eigenvectors of $J_{F}(E)$, which are not parallel to either axis. 
The following picture illustrates the iterates of $F^{2}$ when $\beta_{2}=B_{2}{ }^{2}-B_{2}$.

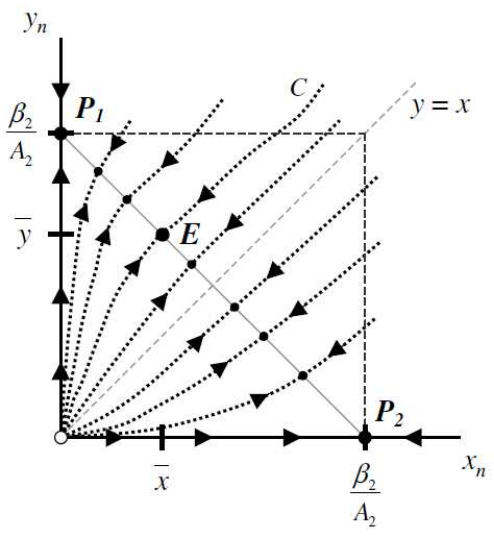

By Theorem 2.4, there is an increasing curve $C$ through $E$ that is invariant under $F^{2}$ and is a subset of the basin of attraction of $E$. As in Region 2 of the parameter space, this curve extends indefinitely to the north-east of $E$ and extends from the south-west of $E$ to the point $(0,0)$, where $F^{2}$ is undefined.

Using the same argument as in Region 2, one can show that in Region 3 of the parameter space, whenever $\left(x_{0}, y_{0}\right)$ is not on curve $C$, the points in the subsequence $\left\{\left(x_{2 n}, y_{2 n}\right)\right\}$ are on one side of curve $C$, while the points in the subsequence $\left\{\left(x_{2 n+1}, y_{2 n+1}\right)\right\}$ are on the other side of curve $C$. Each subsequence converges to a point on line segment (3.10). Thus, the solution of system (3.1) converges to a minimal period-2 point of the system.

\section{Future Research}

In systems of rational difference equations, if an equilibrium point has a stable manifold, it is often a curve whose equation is difficult to determine.

More research can be done to find the equations of the stable manifolds of fixed points of maps that correspond to a system of rational difference equations. This is important research, because in many cases, these equations will give us explicit formulas for the basin of attraction of a fixed point of the map. Additionally, the equations of the invariant curves can sometimes be used to construct an explicit formula for the limit point of a solution of the system of difference equations.

\section{REFERENCES}

[1] E. Camouzis, M. R. S. Kulenović, G. Ladas, and O. Merino, Rational systems in the plane, J. Difference Equ. Appl., 15 (2009), 303-323. 
[2] E. Camouzis and G. Ladas Dynamics of Third-Order Rational Difference Equations; With Open Problems and Conjectures, Chapman \& Hall/CRC Press, 2008.

[3] M. R. S. Kulenović and O. Merino, Global bifurcation for discrete competitive systems in the plane, Discrete Contin. Dyn. Syst. Ser. B, 12 (1) (2009).

[4] M. R. S. Kulenović and O. Merino, Invariant manifolds for competitive discrete systems in the plane, Int. J. Bifurcation Chaos, 20 (8) (2010).

[5] A. Brett, E. Camouzis, G. Ladas, C. Lynd, On the boundedness character of a rational system, J. Numer. Anal. Stoch. Processes, (2009), 1-10.

[6] E. Camouzis, E. Drymonis, and G. Ladas, On the global character of the system $x_{n+1}=\frac{\alpha_{1}}{x_{n}+y_{n}}$ and $y_{n+1}=\frac{\gamma_{2} y_{n}}{B_{2} x_{n}+y_{n}}$, Commun. Appl. Nonlinear Anal., 16 (2009), $41-50$.

[7] E. Camouzis and G. Ladas, Global results on rational systems in the plane, Part I, J. Difference Equ. Appl., 14 (2009), p. 433-458.

[8] D. Clark, M. R. S. Kulenović, and J. F. Selgrade, On a system of rational difference equations, J. Difference Equ. Appl., 11 (2005), 565-580.

[9] J. M. Cushing, Periodically forced nonlinear systems of difference equations, J. Difference Equ. Appl., 3 (1998), 547-561.

[10] E. A. Grove, Y. Kostrov, M. A. Radin, and S. W. Schultz, On the Global Behavior of $x_{n+1}=\frac{\alpha_{1}}{x_{n}+y_{n}}$ and $y_{n+1}=\frac{\alpha_{2}+y_{n}}{B_{2} x_{n}+y_{n}}$, Commun. Appl. Nonlinear Anal., 14 (2007), $35-56$.

[11] M. R. S. Kulenović and M. Nurkanovic, Basins of attraction of an anti-competitive system of difference equations in the plane, Commun. Appl. Nonlinear Anal., 2 (2012), $41-53$.

[12] M. R. S. Kulenović and M. Nurkanovic, Asymptotic behavior of a competitive system of linear fractional difference equations in the plane, J. Inequal. Appl., (2005), 127144.

[13] M. R. S. Kulenović and O. Merino, Competitive exclusion vs. competitive coexistence for systems in the plane, Discrete Contin. Dyn. Syst. Ser. B, 6 (2006), 1141-1156.

[14] M. R. S. Kulenović and O. Merino, A global attractivity result for maps with invariant boxes, Discrete Contin. Dyn. Syst. Ser. B, 6 (2006), 97-110.

[15] E. Magnucka-Blandzi and J. Popenda, On the asymptotic behavior of a rational system of difference equations, J. Difference Equ. Appl., 5 (1999), 271-286.

[16] E. Camouzis, A. Gilbert, M. Heissan, and G. Ladas, On the boundedness character of the system $x_{n+1}=\frac{\alpha_{1}+\gamma_{1} y_{n}}{x_{n}}$ and $y_{n+1}=\frac{\alpha_{2}+\beta_{2} x_{n}+\gamma_{2} y_{n}}{A_{2}+x_{n}+y_{n}}$, Commun. Appl, Nonlinear Anal., (2009), 41-50.

[17] M. R. S. Kulenović and G. Ladas, Dynamics of a Second Order Rational Difference Equation, Chapman \& Hill/CRC Press, (2005).

(Received: May 8, 2012)

University of Rhode Island

Department of Mathematics

Kingston, RI 02881-0816, USA

E-mail: chris@math.uri.edu 